\title{
Health Misinformation
}

\author{
Xiaoli Nan, Yuan Wang, and Kathryn Thier \\ University of Maryland
}

\section{Citation:}

Nan, X., Wang, Y., \& Thier, K. (2021). Health misinformation. In T. Thompson \& N.

Harrington (Eds.), The Routledge Handbook of Health Communication (pp. 318-332).

Routledge. 


\begin{abstract}
Research on health misinformation has grown rapidly as concerns about the potential harmful effects of health misinformation on individuals and society intensify amid a "post-truth" era. In this chapter, we provide a broad overview of current research and evidence concerning the many facets of health misinformation, including its sources, prevalence, characteristics (both content and diffusion features), impact, and mitigation. We conclude that health misinformation originates from many sources, most notably mass and social media; is fairly prevalent, both in interpersonal and mediated settings; and tends to feature negative sentiments, anecdotal evidence, and anti-science narratives. Although there is no conclusive evidence that health misinformation spreads more broadly than scientific information, health misinformation reliably leads to misperceptions on health issues. Efforts to mitigate the impact of health misinformation show early promise in correcting misperceptions. We offer several directions for future research.
\end{abstract} Keywords: misinformation, health, communication, social media 


\section{Health Misinformation}

Reliable health information is crucial to making informed decisions about one's health. Because most of us are not medical experts, we turn to professionals who have the expertise for reliable health information. Indeed, when it comes to health information sources, Americans trust doctors or healthcare professionals the most, followed by government health agencies (Jackson et al., 2019). However, when asked "the most recent time you looked for information about health or medical topics, where did you go first?" more than $72 \%$ of Americans reported "the Internet" (Health Information National Trends Survey [HINTS], 2019). Moreover, 64\% of U.S. adults reported "some" or "a lot" of trust in the Internet as a health information source (HINTS, 2018). The frequency with which the Internet is used as a health information source combined with the level of trust people have in this source is troubling given the prevalence of health misinformation online (Southwell \& Thorson, 2015).

Swire-Thompson and Lazer (2020) define misinformation as "information that is contrary to the epistemic consensus of the scientific community regarding a phenomenon" (p. 434). Health misinformation is a specific type of misinformation that contains one or more healthrelated claims inconsistent with scientific consensus. Ever since the arrival of the Internet, there has been rising concern about online health misinformation and its potential impact on Internet users (Oravec, 2000). In recent years, such concerns have intensified amid the anti-vaccine movement that aggressively utilizes the Internet and social media to spread vaccine misinformation and anti-vaccine sentiment (Kata, 2012). The 2016 U.S. presidential election, which was mired in controversies of political misinformation and foreign interference via social media, allegedly ushered in the "post-truth" era where even health information is being manipulated by foreign entities to stir social discord and polarization. For example, Russian 
Twitter bots have amplified not only anti-vaccine messages but also pro-vaccine content, sowing social discord on a critical public health issue (Broniatowski et al., 2018).

The Internet and social media are not the only sources of health misinformation. Much health misinformation originates from traditional media reports, which then gets amplified on the World Wide Web. In this chapter we provide an overview of prominent sources of health misinformation that include both online and offline sources, followed by a review of evidence concerning the prevalence of health misinformation in diverse settings. We then describe the key characteristics of health misinformation, both in terms of content and diffusion features, as compared to accurate or scientific information. Finally, we review evidence concerning the impact of health misinformation on its receivers and the effectiveness of communication strategies in mitigating the impact of health misinformation.

\section{Sources of Health Misinformation}

Health misinformation comes from many sources, including media, industry, government and politicians, healthcare providers themselves, and social/interpersonal groups or community networks. Within media, the emergence of the Internet has radically changed how health misinformation is generated and distributed, with power shifting to non-experts (Mahoney et al., 2015). Below, we explain how health misinformation is disseminated from each source.

\section{Media}

Perhaps the most common source of health misinformation is the media. Journalistic media may disseminate health misinformation through oversimplification, misrepresentation, and overdramatization as reporters repackage science for a mass audience and adhere to the journalistic principles of "balance" (Lewandowsky et al., 2012, p. 110; Thomas et al., 2017). For instance, the popular press misinforms the public about nutrition by confusing correlation with 
causation, seizing on initial research, and citing non-scientists, such as celebrities and fitness professionals (Ayoob et al., 2002). By engaging in core functions such as surveillance and attracting public attention to gain advertisers, TV reporting can overemphasize or sensationalize novel but rare diseases, such as West Nile virus, ultimately increasing the public's threat perception beyond the actual risk (Gollust et al., 2019). Production and marketplace pressures, along with diminishing resources, can lead TV journalists to oversimplify health research, as can their emphasis on short-form storytelling via stations' social media accounts (Gollust et al., 2019).

In covering health misinformation as news, traditional journalistic media spread falsehoods that are in turn dispersed by Internet news and users. After former U.S. Representative Michele Bachman falsely proclaimed on the Today Show that the HPV vaccine causes mental retardation, an increasing number of Google News and Twitter articles included her inaccurate statement, despite some Twitter users correcting it (Mahoney et al., 2015). Following news reports that Italian courts awarded compensation for alleged MMR-vaccineinduced autism, Google searches, tweets, and Facebook posts about the false connection between vaccines and autism rose, spreading misinformation and anti-vaccination sentiment (Carrieri et al., 2019).

People encounter health misinformation online through searches, content generation, and engagement with mobile health apps (Swire-Thompson \& Lazer, 2020). Sometimes digital tools such as online drug information compendia (Randhawa et al., 2016) and online cancer risk calculators (Levy et al., 2008) contain misinformation even though they are designed to help healthcare providers, patients, or health information consumers. Despite positive outcomes such as patient empowerment (Kata, 2012), the Internet allows non-experts to spread misinformation 
as they create new content in online health forums or communities for people with specific diseases or health concerns (e.g., Bakke, 2019). Additionally, social media can foster "digital pandemics," the widespread online dissemination of medically inaccurate information, including disinformation by anti-vaccine activists who employ interaction and user-generated content to sow apprehension, ambiguity, and misgivings (Seymour et al., 2015, p. 517).

Finally, entertainment media have been a source of health misinformation. Prime time network TV shows and horror films, for example, convey inaccuracies and promote stereotypes about mental illness (Goodwin, 2014). Moreover, medical TV talk shows, which blend facts and entertainment, offer accurate advice only $30 \%$ to $50 \%$ of the time (Thomas et al., 2018).

\section{Industry}

With a decades-long campaign to withhold information about the dangers of smoking and create confusion about the science, the tobacco industry set the standards for industry dissemination of health misinformation (Cappella et al., 2015). In response to legal and government sanctions, the tobacco industry undermines plain packaging rules by replacing banned words ("mild" and "light") with other misleading ones ("smooth") and choosing lighter colors for brand names to reinforce misperceptions that "light" cigarettes are less harmful (Evans-Reeves et al., 2019). The global, industrialized food industry has adapted the tobacco industry's earlier tactics by advertising non-nutritious food to children, promoting conflicting data about fats and sugars, and employing misleading words such as "natural" and "light" to create health claims unsupported by science (Hindin et al., 2004). Similarly, the limited information conveyed in direct-to-consumer pharmaceutical advertisements of complex and nuanced topics, such as genetic testing and specialized cancer center treatment, can lead to misperceptions (Hlubocky et al., 2020). 


\section{Government and Politicians}

Exploiting vaccine hesitancy is now part of the arsenal of populist political discourse, with government-sponsored Russian Twitter trolls seeding debate to undermine the 2016 U.S. elections (Broniatowski et al., 2020) and Polish right-wing politicians aligning with anti-vaccine activists as they construct a nationalist populist narrative (Żuk et al., 2019). In the United States, politicians on the right, including the former U.S. president Donald Trump, have spread misinformation about oral contraceptives to attack the Affordable Care Act (Hogue et al., 2017), vaccines (Mahoney et al., 2015), and the emergence of COVID-19 and possible treatments (Jaiswal et al., 2020).

\section{Healthcare Providers}

Providers, including doctors, nurses, dental hygienists, medical students, and pharmacists, spread misinformation to patients in various ways. For example, they may have inadequate knowledge (e.g. Clovis et al., 2012). They also may adhere to false beliefs prevalent in their specialty, such as psychiatrists who discount the evidence that antidepressants create physical dependence and significant, common withdrawal symptoms (Hengartner \& Plöderl, 2018). In addition, some providers may rely on unsound clinical evidence (Ioannidis et al., 2017) or literature tainted by citation bias, as when studies about the high prevalence of smoking among schizophrenics are over-cited despite an average smoking prevalence in that population (Chapman et al., 2009). As with non-professionals, practitioners also disseminate and consume misinformation via social media (Chua \& Banerjee, 2018).

\section{Informal Groups/Community Networks}

Informal networks, in-group attitudes, and rumors often serve as health misinformation sources in communities with a history of medical mistrust (DiFonzo et al., 2012). For instance, 
conspiracy theories thrive in situations of "inequality-driven mistrust" (Jaiswal et al., 2020, p. 1), such as HIV/AIDS conspiracy narratives among some African Americans (Heller, 2015). Rumors tend to circulate through "trust and knowledge networks" created among ethnic or racial subgroups due to social constraints (Heller, 2015, p. 43). Several studies document how women in developing countries or who are low-income learn health misinformation from interpersonal rumors, including about contraception among women in India, Nepal, and Nigeria (DiamondSmith et al., 2012). Also, cultural reliance on word-of-mouth and informal networks likely led to health misinformation among African American cancer patients (Matthews et al., 2002), MMR vaccine-hesitant Somali Americans (Bahta \& Ashkir, 2015), and Latinas with respect to sexual health (Cashman et al., 2011).

\section{Summary}

Sources of health misinformation are largely similar to general misinformation sources. The ability of non-experts to create and spread misinformation via online user-generated content and social media is shifting the landscape of health misinformation sources. Informal groups and community networks, such as those derived from race, ethnic, and gender identities, can be misinformation sources and warrant more systematic investigation, as does the interplay between these offline and online networks.

\section{Prevalence of Health Misinformation}

We are concerned about health misinformation because we believe it is prevalent and has the potential of reaching large audiences. So, how prevalent is health misinformation according to past research? And in what communication settings is health misinformation more prevalent? Many studies have examined the prevalence of health misinformation in such diverse contexts as tobacco products (Evans-Reeves et al., 2019), cancer (Chen et al., 2018), immunization 
(Goodyear-Smith et al., 2007; Thomas et al., 2017), and infectious diseases (Kouzy et al., 2020).

These studies provided insights regarding the prevalence of health misinformation in interpersonal and mediated communication settings.

\section{Interpersonal Settings}

People often rely on informal and familial word-of-mouth channels for health advice, especially when formal sources of information, such as the medical community, are distrusted (DiFonzo et al., 2012). Anderson et al. (2014) interviewed a diverse sample of women regarding how they communicate information about contraceptives with others in their social networks. Participants reported that information about negative aspects of contraceptive methods, which often was incorrect, was both more prevalent and more memorable than positive information about contraceptive methods in their communication with friends and family members. In another study about cancer misinformation, DiFonzo et al. (2012) had participants recall a statement they heard about cancer from a nonmedical source. Results showed that around $83 \%$ of these statements were rumors (i.e., unverified statements in circulation) and $85 \%$ of the rumors came from friends or family. These findings suggest that health misinformation likely is quite common in interpersonal settings, especially in communication with close social ties.

Health misinformation also occurs in patient-provider communication. In a study assessing the prevalence of misinformation about emergency contraception in communication between pharmacists and adolescents, Wilkinson et al. (2012) telephoned 759 pharmacies in five U.S. cities while posing as 17-year-old adolescents. They found that around one in five pharmacies spread misinformation regarding who can take emergency contraception and almost half of pharmacies communicated incorrect age guidelines to callers. In another study of patients' postpartum contraception perceptions, Sundstrom et al. (2018) found that although 
participants overall demonstrated trust in their healthcare provider's advice, some patients also mentioned receiving conflicting advice and misinformation regarding risks of contraception from their providers. Provider recommendations are among the most important information patients refer to when making health decisions (Anderson et al., 2014). Therefore, any health misinformation circulated in patient-provider communication, regardless of prevalence, raises serious concerns.

\section{Mediated Settings}

Although misinformation does occur and can be fairly prevalent in non-mediated, interpersonal communication, misinformation is often considered a mass communication phenomenon (Southwell \& Thorson, 2015). Fear of misinformation in mass media likely stems from perceived ability of the mass media to quickly and widely spread misinformation. In general, research has shown misinformation to be prevalent in the mass media, including traditional media and especially online media.

Journalists for traditional media outlets perform a gatekeeping function, yet research has found that misinformation is still a serious problem in traditional media. In a study concerning the prevalence of vaccine misinformation in media reports, Goodyear-Smith et al. (2007) analyzed the content of 400 national publications in 2001 and in 2003 throughout New Zealand. They found that $17 \%$ of the articles contained anti-immunization messages, which were seldom backed up by scientific evidence. In another study examining how news and public affairs articles reported on the false statement "HPV vaccine causes mental retardation," Thomas et al. (2017) found that about half of these articles included the statement without explicitly stating the claim was false.

The increasing popularity of social media as a venue for seeking and sharing health 
information amplifies the prevalence of health misinformation on social media (Southwell \& Thorson, 2015). In a study of medical misinformation pertaining to the COVID-19 pandemic on Twitter, Kouzy et al. (2020) examined 673 tweets related to the virus; they found that 153 tweets (24.8\%) included misinformation and 107 tweets (17.4\%) included unverifiable information related to the pandemic. In another study of psoriasis-related misinformation on YouTube, Qi et al. (2016) analyzed the content of 47 videos and found that $21 \%$ of these videos were misleading, containing inaccurate or unproven information about psoriasis based on current evidence. Health misinformation was also found to be prevalent on other social media platforms, including Facebook (Jamison et al., 2020), WhatsApp (Al Khaja et al., 2018), and Weibo (Chen et al., 2018). These findings highlight the importance of curbing the dissemination of health misinformation on social media.

\section{Summary}

A wealth of studies has examined the prevalence of health misinformation in various communication settings. Although the specific proportion of misinformation varies across health contexts and communication settings, health misinformation overall appears to be quite common in both interpersonal and mediated communication.

\section{Characteristics of Health Misinformation}

Although evidence is growing on the prevalence of health misinformation, less is known about the characteristics of health misinformation. Examining how health misinformation differs from scientific information in terms of content characteristics (Moran et al., 2016; Zollo et al., 2015) and diffusion patterns (Chen et al., 2018; Kouzy et al., 2020) is an emerging trend.

\section{Content Characteristics}

How are the content characteristics of health misinformation different from those of 
typical scientific information? Current research suggests differences in at least two areassentiment and evidence. Moreover, health misinformation is reliant on an anti-science rhetoric and is often misleading rather than ostensibly false.

In terms of sentiment, health misinformation tends to be more negative in tone than scientific messages. In a study examining word usage and sentiment differences between proand anti-vaccine headlines of online articles, Xu and Guo (2018) found that anti-vaccine headlines were 1.3 times more likely to use words related to negative sentiments, including fear, anger, and sadness, than pro-vaccine headlines. In another study that analyzed the sentiments of a collection of conspiracy posts and scientific news posts on Facebook, Zollo et al. (2015) drew a similar conclusion; they found that $54 \%$ of conspiracy posts used negative sentiment, double the amount in scientific ones.

Regarding the evidence underlying the claims of the message, health misinformation is often based on anecdotes and personal stories, whereas scientific messages are usually backed up by facts and statistics (Teoh, 2019). For instance, in a content analysis of anti-vaccine websites, Moran et al. (2016) found that $66.9 \%$ of these websites used pseudo-scientific evidence (e.g., confusing correlation for causation) and $30.6 \%$ of them used anecdotal evidence to support their claims. Instead of emphasizing the scientific evidence, these websites promoted anti-vaccine statements by connecting anti-vaccine behavior to various values, such as choice, individuality, and freedom. In another study of vaccine misinformation, Grant et al. (2015) analyzed the content of two pro-vaccine and two vaccine-skeptical websites; they found that pro-vaccine websites concentrated on conveying evidence-based scientific research about vaccines, whereas vaccine-skeptical websites focused more on creating communities of people affected by vaccinerelated practices. 
With regard to rhetorical tactics, studies show that health misinformation often uses antiscience narratives. In a study of vaccine misinformation on social media, Steffens et al. (2019) found that the misinformation spread by anti-vaccine activists was characterized by resistance to mainstream expertise and skepticism of scientific evidence. In another study of anti-vaccination movements, Kata (2012) identified three tactics that anti-vaccine activists used to argue against vaccination: skewing science, shifting hypotheses, and attacking critics. The author concluded that these tactics make vaccine misinformation look convincing despite lacking scientific support.

Moreover, misinformation is not always easy to discern. Past research has tended to focus on misinformation that is unequivocally false, whereas real-world misinformation can be misleading in subtle ways, with misdirection resulting solely from hints, framing, word choices, and relative placement of various pieces of information (Ecker et al., 2014). In a content analysis of drug-related messages on social media, Al Khaja et al. (2018) found that potentially misleading claims (i.e., claims that omit important facts about the drug, use poor-quality clinical evidence as scientific support, or exaggerate the clinical importance of poor-quality evidence) comprised the majority of the messages $(59.1 \%)$, more than twice the amount of false claims $(27.3 \%)$ that directly contradict evidence or lack any supporting evidence. In sum, the most prominent types of misinformation in real-life settings might be misleading claims rather than explicit falsehoods.

\section{Diffusion Patterns}

Do lies travel faster than truths, as people have lamented? It sure does seem so in a study conducted by Vosoughi et al. (2018), who analyzed 126,000 news stories circulated on Twitter from 2006 to 2017 and found that misinformation spread significantly farther, faster, deeper, and 
more broadly than did accurate information. The authors posited that misinformation spread more widely than facts because it elicited more high-arousal emotions such as disgust, fear, and surprise, which motivated the recipients to share the misinformation. In a specific health context, $\mathrm{Xu}$ and Guo (2018) found that anti-vaccine information was shared, commented on, and liked more than pro-vaccine information.

Other studies have provided contrary evidence, however. In a recent analysis of medical misinformation in the COVID-19 pandemic on Twitter, Kouzy et al. (2020) found that neither false claims nor unverifiable information received a significantly different number of likes and retweets than scientific claims, suggesting that misinformation is as likely to spread as truthful information. In a network analysis of cancer-related misinformation, Chen et al. (2018) analyzed the diffusion pattern of cancer-related misinformation on Weibo, a popular Chinese social media platform similar to Twitter. They found that although several false tweets had been extremely popular and received a large number of retweets, most of the false tweets received fewer retweets and comments than truthful tweets; in addition, truthful tweets showed better diffusion performance in terms of range and structural virality of retweet networks. These findings suggest that although a few pieces of misinformation could be extremely viral, scientific messages may have a better diffusion performance overall.

\section{Summary}

Health misinformation is characterized by negative sentiments, anecdotal evidence, and anti-science narratives. It is often subtly misleading rather than explicitly false. There are mixed findings regarding whether health misinformation diffuses more broadly than scientific messages. We need more studies, in more diverse health and media platform contexts, to further evaluate the diffusion characteristics of misinformation versus accurate information. 


\section{Impact of Health Misinformation}

Although misinformation has always been a significant part of human communication, the unprecedented proliferation of misinformation in general and health misinformation in particular on social media and other forms of online and traditional media in recent years has led to grave concerns among conscientious citizens, scientists, and government officials (Chou et al., 2018; Southwell \& Thorson, 2015). The concerns rest on the assumption that misinformation has the potential to significantly impact how people think, feel, and behave with regard to the issues tainted with misinformation, undermining the integrity of democracy and jeopardizing public health. In the political arena, for example, it is believed that online misinformation propagated by Russian trolls influenced the outcome of the 2016 American presidential election (Jamieson, 2018). In the public health domain, recent measles outbreaks in the United States have been attributed to misinformation about vaccine safety (Centers for Disease Control and Prevention, 2019).

Despite the high level of concern about online misinformation and its potential effects, empirical evidence regarding the impact of misinformation remains limited. In a study of fake news sharing by registered voters on Twitter during the 2016 presidential election, Grinberg et al. (2019) found that engagement with fake news sources was extremely concentrated; only $1 \%$ of individuals accounted for $80 \%$ of fake news source exposures, and $0.1 \%$ accounted for nearly $80 \%$ of fake news sources shared. Tracking visits to fake news websites, Guess et al. (2020a) showed that these sites made up a small share of people's information diets on average and were largely consumed by a subset of Americans with strong preferences for pro-attitudinal information. These findings suggest that fake news or political misinformation may have had less impact on the election outcome than previously assumed. On the basis of emerging correlational 
and experimental evidence concerning the effects of misinformation exposure in political contexts, Guess et al. (2020b) concluded that fake news may have limited effects beyond increasing beliefs in false claims and that many claims about the effects of exposure to false news may be overstated.

What about health misinformation? To what extent are people's health beliefs, attitudes, and even their behaviors influenced by health misinformation? Given the widespread concern about the potential detrimental impact of health misinformation (Swire-Thompson \& Lazer, 2020), there is surprisingly limited research directly examining such impact, and the evidence that is available so far appears to be mixed. In a longitudinal study examining the lagged associations between young adults' exposure to misinformation about cancer-related risks (e.g., indoor tanning, e-cigarette use, reuse of plastic bottles, and use of artificial sweetener) and related beliefs, intentions, and behaviors, Tan et al. (2015) found that exposure to misinformation predicted misperceptions for only one of the four contexts examined; people who reported more exposure to the false statement that reusing plastic bottles can cause cancer were more likely to believe it is true. Believing in this false statement was inconsequential to reported intentions or behaviors, however.

In the context of vaccination, Smith et al. (2008) found that news media coverage of the MMR-autism controversy following the publication of the 1998 Lancet article linking MMR vaccination to childhood autism bore no relationship with the rate of selective MMR nonreceipt (cases where children received all childhood immunizations except MMR) in the United States. This finding suggests that news media coverage of vaccine misinformation may have limited influence on parental decisions to vaccinate their children, but it cannot rule out the potential impact of vaccine misinformation distributed in other media platforms such as social media. 
Edelstein et al. (2020) examined childhood vaccine coverage in the United Kingdom and antivaccination sentiment on Twitter. They found that vaccination coverage among children had been in decline since 2012-2013 even though there was a decrease in the proportion of antivaccination messages on Twitter. These findings show that online anti-vaccination messages might have limited impact on vaccination behavior. The authors warned that exaggerating the potential impact of the anti-vaccination movement may bring more attention to anti-vaccination messages that health professionals wish parents to avoid in the first place.

The impact of health misinformation can be more convincingly demonstrated in experimental studies where causal inferences can be made. In an experimental study of tobacco misinformation, for example, Gratale et al. (2019) exposed current and former smokers to cigarette ads that contained misleading claims (e.g., "100\% additive free and U.S. grown - no chemicals, flavourings or preservatives...," p. e44). Compared to participants who saw a similar ad with the misleading words (e.g., "additive free," "no chemical," p. e44) removed, those exposed to the misleading ad reported stronger misperceptions about the tobacco product and, among current smokers, greater intention to use it. These findings suggest that misinformation can lead to misperception and change in behavioral intention.

In another experimental investigation, Lyons et al. (2019) had participants read information about the Zika virus with or without a conspiracy theory (i.e., the Zika epidemic was the result of the release of genetically modified mosquitoes by a pharmaceutical company that would profit from the need for a Zika vaccine). They found that exposure to the information containing a conspiracy theory significantly increased participants' conspiracy beliefs. They also tested the impact of the explicitness of conspiracy cues (i.e., whether the conspiracy theory was explicitly stated or merely suggested) and found that misinformation with 
explicit conspiracy cues resulted in greater conspiracy beliefs. Yet, misinformation did not have a tangible impact on behavioral intentions (i.e., intentions to uptake the Zika vaccine).

\section{Summary}

Overall, health misinformation significantly influences health beliefs, often leading to misperceptions. Evidence concerning the influence of exposure to health misinformation on other outcome variables such as attitudes, behavioral intentions, or behaviors is limited, and the findings tend to be mixed.

\section{Mitigation of the Impact of Health Misinformation}

In the past few years, we have seen a rapid expansion of research on mitigating the impact of misinformation in health and other contexts, fueled by the pressing concern about the potential negative impact of misinformation. Numerous studies emerged that explored the efficacy of corrective messages in reducing misperceptions in general (for reviews see Walter \& Murphy, 2018; Walter \& Tukachinsky, 2020) and health misperceptions in particular (Bode \& Vraga, 2018; Lee et al., 2020). Other studies have examined ways to build resistance to misinformation through pre-interventions such as forewarning (Cook et al., 2017) and innovative education (Roozenbeek \& van der Linden, 2019).

\section{Misperception Correction}

Given the prevalence of vaccine misinformation in the social media space, several studies examined the effectiveness of corrective messages in changing vaccine misperceptions. For example, Nyhan et al. (2014) studied a national sample of parents and exposed them randomly to one of several pro-MMR vaccine messages or no information at all. Among the pro-MMR vaccine messages was a corrective message that presented scientific evidence debunking the vaccine/autism link. The authors found that the corrective message successfully reduced belief in 
the vaccine/autism link but did not influence overall concern about the side effects of the MMR vaccine. The corrective message did not change parents' intention to give a future child the MMR vaccine. Among parents who held the least favorable initial attitudes toward vaccines, the corrective message actually backfired by decreasing these parents' intentions to vaccinate their children. The backfire or boomerang effect was similarly observed in a study on flu vaccination, where participants with high levels of concern about vaccine side effects decreased their intent to get the flu vaccine after viewing a message debunking a flu vaccine myth (that the flu vaccine gives one the flu; Nyhan \& Reifler, 2015). Although the backfire effects of debunking messages on vaccination intentions are disconcerting, future research is needed to examine the extent of this phenomenon across different health behaviors. A recent study examining backfire effects of debunking messages on belief change in various contexts of political issues suggests that the backfire effect might be an exception rather than a rule (Wood \& Porter, 2019).

Other health contexts that have received considerable attention in terms of misinformation debunking and misperception correction include tobacco products (Sangalang et al., 2019) and infectious diseases such as the Zika virus (Bode \& Vraga, 2018; Lyons et al., 2019). In addition to examining the general effects of corrective messages, studies have assessed the effects of corrective messages as a function of message type (Sangalang et al., 2019), source of the message (Pluviano et al., 2020; Vraga \& Bode, 2017), and audience characteristics (Nyhan et al., 2014).

In terms of message type, Vraga et al. (2019), for example, compared the efficacy of logic-based versus humor-based corrective messages in changing misperceptions about three science or political topics (climate change, gun control, and HPV vaccination). The authors found that logic-based corrective messages were consistently effective across topics, whereas 
humor-based corrective messages were effective only in correcting misperceptions about HPV vaccination. For HPV vaccination, logic-based messages also performed better than humor-based messages in correcting misperceptions.

In terms of message source, Vraga and Bode (2017) found that corrective messages delivered by a known credible source (e.g., Centers for Disease Control and Prevention) were more effective in reducing misperceptions about the Zika virus than those posted by an unknown user. Pluviano et al. (2020) further demonstrated that when it comes to correcting health misperceptions (e.g., belief in a rumored link between a vaccine and ADHD), source trustworthiness is more important than source expertise; sources high (vs. low) in trustworthiness were found to be more effective in reducing the use of misinformation in inferences, whereas source expertise did not make a difference.

Perhaps the most common audience characteristic researched in health misperception correction studies is individuals' initial belief in misinformation. Because individuals tend to be more accepting of cognitively consistent information and more skeptical of inconsistent information - a phenomenon called motivated reasoning where motivation (to reach a particular conclusion) affects reasoning through reliance on a biased set of cognitive processes (Kunda, 1990) — there is reason to believe that corrective messages are less effective the more people believe in the misinformation in the first place. Empirical evidence tends to be mixed, however (Lewandowsky et al., 2012). In health domains, corrective messages either have similar effects on beliefs for people high and low in initial misperceptions (Nyhan et al., 2014; Nyhan \& Reifler, 2015) or tend to work more effectively for those high in initial misperceptions (Vraga \& Bode, 2017). In the latter case, the lack of effects among those low in initial misperceptions might be due to a floor effect, where the ability of corrective messages to further reduce 
misperceptions could be diminished.

\section{Pre-Interventions}

Through a meta-analysis, Walter and Murphy (2018) found that in general, forewarning is not as effective as debunking or misperception correction in mitigating the impact of misinformation. However, studies show that forewarning could be a useful complementary strategy. In the context of climate change, for instance, Cook et al. (2017) found that preemptively explaining the potentially misleading effect of false-balance media coverage or conveying scientific consensus was effective in neutralizing the negative influence of misinformation on climate change perceptions. Further, innovative educational tools such as a "fake news game" confer psychological resistance against online misinformation (Roozenbeek \& van der Linden, 2019).

\section{Summary}

In sum, important work is being carried out to evaluate the efficacy of corrective messages and pre-interventions in combatting health misinformation. The good news is that corrective messages tend to work well to reduce health misperceptions, at least in the short term. It is less known whether the effects might be long-lasting given the lack of longitudinal studies. We also know that the efficacy of corrective messages may vary as a function of the type of sources, formats, and recipients of the messages. Further, messages correcting vaccine misperceptions seem to have a worrisome backfire effect on vaccine-hesitant parents' intention to vaccinate their children. More research is needed to assess potential backfire effects in other health contexts. Finally, pre-interventions are promising strategies for mitigating the impact of health misinformation, although research on pre-interventions in health contexts is sparse.

\section{General Conclusions}


Health misinformation is a serious threat to public health and warrants systematic research into its sources, prevalence, characteristics, impact, and mitigation. Although current research on health misinformation is still largely descriptive in nature, recent work has begun to use experimental methods to document the impact and mitigation of health misinformation.

Overall, researchers have taken a predominantly empirical, positivistic approach to investigating the various facets of health misinformation. Future research could incorporate more interpretive, critical-cultural perspectives to bring about a more complete understanding of a phenomenon as complex as misinformation in health contexts.

There are a few general conclusions that can be drawn based on the current landscape of the health misinformation literature. First, health misinformation comes from many sources, including personal social networks, industries, government and politicians, and even providers themselves. Perhaps the most common source, however, is the media. Health misinformation reported through traditional journalistic media is amplified by online and social media, where health misinformation increasingly is disseminated through user-generated content.

Second, health misinformation is prevalent in our information environment, both in interpersonal and mediated settings. The overall prevalence of health misinformation presents a significant challenge not only for consumers to obtain accurate and useful health information but also for providers in their interaction with misinformed patients. Third, health misinformation is characterized by negative sentiments, anecdotal evidence, and anti-science narratives. It is often subtly misleading rather than explicitly false, making it challenging to detect or counteract. Although there is concern that misinformation may reach more people than accurate or scientific information, there is limited evidence that health misinformation diffuses more broadly than scientific messages. 
Finally, although health misinformation has been shown to result in health misperceptions, evidence concerning the influence of exposure to health misinformation on other outcome variables such as attitudes, behavioral intentions, or behaviors is limited and mixed. Efforts to mitigate the impact of health misinformation through corrective messages are well under way. Corrective messages tend to work well to reduce health misperceptions, at least in the short term. Their long-term effects and impact on behaviors are less understood, however.

\section{Directions for Future Research}

Future research should continue to monitor the sources of health misinformation. Usergenerated content in online and social media is becoming a common and worrisome source of health misinformation. Such content can originate from individuals or organized groups (e.g., anti-vaccine activists). As access to digital and mobile information sources increases, more research is needed to investigate how online users create and disseminate health misinformation, including the techniques used to increase influence and reach. Another area that needs more attention is misleading advertising and marketing by the food, alcohol, and tobacco industries. The "next frontier on misinformation and smoking" is the recent emergence of vaping and ecigarette use, with accurate information hampered by conflicting views on safety, inconsistent regulations, and questionable YouTube videos and social media ads (Krishna \& Thompson, 2019, p. 8).

More research is needed to understand the exposure to and consumption of health misinformation. What types of individuals are more likely to be exposed to and consume health misinformation and therefore are more vulnerable to its detrimental impact? Older adults or people with lower media literacy, for example, could consume a larger quantity of health misinformation or be targeted disproportionally with misleading health information (e.g., online 
direct-to-consumer pharmaceutical advertising). Identifying vulnerable groups will inform future interventions to reduce exposure and consumption of health misinformation.

Future research should continue to investigate the content and diffusion characteristics of health misinformation as qualified by other aspects of the misinformation. For instance, although negative sentiments are often used in health misinformation (Xu \& Guo, 2018; Zollo et al., 2015), in certain health contexts (e.g., e-cigarettes; Martinez et al., 2018) positive sentiments tend to be more common. Additionally, content characteristics might differ by source. For example, activists often use personal stories and anti-science narratives to support their false claims (Steffens et al., 2019), whereas industries are more likely to present misinformation in a subtle way, often by omitting important facts or overstating the efficacy of products (Al Khaja et al., 2018). Furthermore, a fruitful avenue for future research is to examine the linkage between misinformation's content/source and diffusion characteristics. Do certain features of misinformation make it more viral? Does misinformation from certain sources spread more widely than that from other sources? Studies have found that health messages that presented controversial, emotionally evocative, and familiar content are more likely to go viral (Kim, 2015); however, it is unclear whether the same applies to the virality of health misinformation.

Finally, an important direction for future research is to document the impact of health misinformation. So far empirical evidence shows health misinformation influences health beliefs and misperceptions, but its impact on attitudinal and behavioral outcomes is not well-understood. To what extent does health misinformation lead to actual harm beyond simply implanting bad ideas? It seems intuitive to assume bad ideas or beliefs will eventually lead to harmful behaviors. Until more empirical evidence supports such a link, however, it remains inconclusive. On the other hand, there is a growing interest in evaluating the efficacy of corrective messages in 
mitigating the impact of health misinformation. Research suggests that the efficacy of corrective messages varies widely depending on the characteristics of such messages (e.g., pre-bunking vs. debunking, fact checking vs. alternative explanation). More research is needed to identify factors that strengthen or weaken the effects of corrective messages. Finally, just as there is no robust evidence that health misinformation leads to harmful behavior, efforts to mitigate the impact of health misinformation have not been empirically linked to reduction in harmful behavior. Clearly more work is needed to investigate the relationships between exposure to health misinformation or corrective messages and behavioral outcomes. 


\section{References}

Al Khaja, K. A. J., AlKhaja, A. K., \& Sequeira, R. P. (2018). Drug information, misinformation, and disinformation on social media: A content analysis study. Journal of Public Health Policy, 39(3), 343-357.

Anderson, N., Steinauer, J., Valente, T., Koblentz, J., \& Dehlendorf, C. (2014). Women's social communication about IUDs: A qualitative analysis. Perspectives on Sexual and Reproductive Health, 46(3), 141-148.

Ayoob, K.T., Duyff, R. L., \& Quagliani, D. (2002). Position of the American dietetic association: Food and nutrition misinformation. Journal of the American Dietetic Association, 102(2), 260-266.

Bahta, L., \& Ashkir, A. (2015). Addressing MMR vaccine resistance in Minnesota's Somali community. Minnesota Medicine, 98(10), 33-36.

Bakke, A. (2019). Trust-building in a patient forum: The interplay of professional and personal expertise. Journal of Technical Writing \& Communication, 49(2), 156-182.

Bode, L., \& Vraga, E. K. (2018). See something, say something: Correction of global health misinformation on social media. Health Communication, 33(9), 1131-1140.

Broniatowski, D. A., Jamison, A. M., Qi, S., AlKulaib, L., Chen, T., Benton, A., Quinn, S. C., \& Dredze, M. (2018). Weaponized health communication: Twitter bots and Russian trolls amplify the vaccine debate. American Journal of Public Health, 108(10), 1378-1384.

Broniatowski, D. A., Quinn, S. C., Dredze, M., \& Jamison, A. M. (2020). Vaccine communication as weaponized identity politics. American Journal of Public Health, 110(5), 617-618. 
Cappella, J. N., Maloney, E., Ophir, Y., \& Brennan, E. (2015). Interventions to correct misinformation about tobacco products. Tobacco Regulatory Science, 1(2), 186-197.

Carrieri, V., Madio, L., \& Principe, F. (2019). Vaccine hesitancy and (fake) news: Quasiexperimental evidence from Italy. Health Economics, 28(11), 1377-1382.

Cashman, R., Eng, E., Simán, F., \& Rhodes, S. D. (2011). Exploring the sexual health priorities and needs of immigrant Latinas in the southeastern United States: A community-based participatory research approach. AIDS Education \& Prevention, 23(3), 236-248.

Centers for Disease Control and Prevention. (2019, April 26). CDC Media Statement: Measles cases in the U.S. are highest since measles was eliminated in 2000. https://www.cdc.gov/media/releases/2019/s0424-highest-measles-cases-sinceelimination.html

Chapman, S., Ragg, M., \& McGeechan, K. (2009). Citation bias in reported smoking prevalence in people with schizophrenia. Australian \& New Zealand Journal of Psychiatry, 43(3), $277-282$.

Chen, L., Wang, X., \& Peng, T. Q. (2018). Nature and diffusion of gynecologic cancer-related misinformation on social media: Analysis of tweets. Journal of Medical Internet Research, 20(10), e11515.

Chou, W. Y. S., Oh, A., \& Klein, W. M. (2018). Addressing health-related misinformation on social media. JAMA: Journal of the American Medical Association, 320(23), 2417-2418.

Chua, A. Y. K., \& Banerjee, S. (2018). Intentions to trust and share online health rumors: An experiment with medical professionals. Computers in Human Behavior, 87, 1-9.

Clovis, J. B., Horowitz, A. M., Kleinman, D. V., Wang, M. Q., \& Massey, M. (2012). Maryland dental hygienists' knowledge, opinions and practices regarding dental caries prevention 
and early detection. Journal of Dental Hygiene, 86(4), 292-305.

Cook, J., Lewandowsky, S., \& Ecker, U. K. (2017). Neutralizing misinformation through inoculation: Exposing misleading argumentation techniques reduces their influence. PloS One, 12(5), e0175799.

Diamond-Smith, N., Campbell, M., \& Madan, S. (2012). Misinformation and fear of side-effects of family planning. Culture, Health \& Sexuality, 14(4), 421-433.

DiFonzo, N., Robinson, N. M., Suls, J. M., \& Rini, C. (2012). Rumors about cancer: Content, sources, coping, transmission, and belief. Journal of Health Communication, 17(9), $1099-1115$.

Ecker, U. K., Lewandowsky, S., Chang, E. P., \& Pillai, R. (2014). The effects of subtle misinformation in news headlines. Journal of Experimental Psychology: Applied, 20(4), $323-325$.

Edelstein, M., Müller, M., Ladhani, S., Yarwood, J., Salathé, M., \& Ramsay, M. (2020). Keep calm and carry on vaccinating: Is anti-vaccination sentiment contributing to declining vaccine coverage in England? Vaccine. Advance online publication.

Evans-Reeves, K. A., Hiscock, R., Lauber, K., \& Gilmore, A. B. (2019). Prospective longitudinal study of tobacco company adaptation to standardised packaging in the UK: Identifying circumventions and closing loopholes. BMJ Open, 9(9), e028506.

Gollust, S. E., Fowler, E. F., \& Niederdeppe, J. (2019). Television news coverage of public health issues and implications for public health policy and practice. Annual Review of Public Health, 40, 167-185.

Goodwin, J. (2014). The horror of stigma: Psychosis and mental health care environments in twenty-first-century horror film (part II). Perspectives in Psychiatric Care, 50(4), 224 
234.

Goodyear-Smith, F., Petousis-Harris, H., Vanlaar, C., Turner, N., \& Ram, S. (2007).

Immunization in the print media - perspectives presented by the press. Journal of Health Communication, 12(8), 759-770.

Grant, L., Hausman, B. L., Cashion, M., Lucchesi, N., Patel, K., \& Roberts, J. (2015).

Vaccination persuasion online: A qualitative study of two pro-vaccine and two vaccineskeptical websites. Journal of Medical Internet Research, 17(5), e133.

Gratale, S. K., Maloney, E. K., \& Cappella, J. N. (2019). Regulating language, not inference: An examination of the potential effectiveness of Natural American Spirit advertising restrictions. Tobacco Control, 28(e1), e43-e48.

Grinberg, N., Joseph, K., Friedland, L., Swire-Thompson, B., \& Lazer, D. (2019). Fake news on Twitter during the 2016 US presidential election. Science, 363(6425), 374-378.

Guess, A. M., Nyhan, B., \& Reifler, J. (2020a). Exposure to untrustworthy websites in the 2016 US election. Nature Human Behaviour, 4(5), 472-480.

Guess, A. M., Lockett, D., Lyons, B., Montgomery, J. M., Nyhan, B., \& Reifler, J. (2020b).

"Fake news" may have limited effects beyond increasing beliefs in false claims. Harvard Kennedy School Misinformation Review, 1(1), 1-12.

Health Information National Trends Survey. (2018). HINTS Briefs. https://hints.cancer.gov/docs/ Briefs/HINTS_Brief_39.pdf

Health Information National Trends Survey. (2019). HINTS Response. https://hints.cancer.gov/ view-questions-topics/question details.aspx?PK_Cycle=12\&qid=688

Heller, J. (2015). Rumors and realities: Making sense of HIV/AIDS conspiracy narratives and contemporary legends. American Journal of Public Health, 105(1), e43-e50. 
Hengartner, M. P., \& Plöderl, M. (2018). False beliefs in academic psychiatry: The case of antidepressant drugs. Ethical Human Psychology \& Psychiatry, 20(1), 6-16.

Hindin, T. J., Contento, I. R., \& Gussow, J. D. (2004). A media literacy nutrition education curriculum for head start parents about the effects of television advertising on their children's food requests. Journal of the American Dietetic Association, 104(2), 192-198.

Hlubocky, F. J., McFarland, D. F., Spears, P. A., Smith, L., Patten, B., Peppercorn, J., \& Holcombe, R. (2020). Direct-to-consumer advertising for cancer centers and institutes: Ethical dilemmas and practical implications. American Society of Clinical Oncology Educational Book, 40, e207-e217.

Hogue, C. J., Hall, K. S., \& Kottke, M. (2017). Hormonal contraceptives improve women’s health and should continue to be covered by health insurance plans. Annals of Internal Medicine, 167(9), 666-667.

Ioannidis, J. P. A., Stuart, M. E., Brownlee, S., \& Strite, S. A. (2017). How to survive the medical misinformation mess. European Journal of Clinical Investigation, 47(11), 795802.

Jackson, D. N., Peterson, E. B., Blake, K. D., Coa, K., \& Chou, W.-Y. S. (2019). Americans' trust in health information sources: Trends and sociodemographic predictors. American Journal of Health Promotion, 33(8), 1187-1193.

Jaiswal, J., LoSchiavo, C., \& Perlman, D. C. (2020). Disinformation, misinformation and inequality-driven mistrust in the time of COVID-19: Lessons unlearned from AIDS denialism. AIDS and Behavior. Advance online publication.

Jamieson, K. H. (2018). Cyberwar: How Russian hackers and trolls helped elect a president: What we don't, can't, and do know. Oxford University Press. 
Jamison, A. M., Broniatowski, D. A., Dredze, M., Wood-Doughty, Z., Khan, D., \& Quinn, S. C. (2020). Vaccine-related advertising in the Facebook Ad Archive. Vaccine, 38(3), 512 520.

Kata, A. (2012). Anti-vaccine activists, Web 2.0, and the postmodern paradigm - An overview of tactics and tropes used online by the anti-vaccination movement. Vaccine, 30(25), 3778-3789.

Kim, H. S. (2015). Attracting views and going viral: How message features and news-sharing channels affect health news diffusion. Journal of Communication, 65(3), 512-534.

Kouzy, R., Abi Jaoude, J., Kraitem, A., El Alam, M. B., Karam, B., Adib, E., Zarka, J., Traboulsi, C., Akl, E.W., \& Baddour, K. (2020). Coronavirus goes viral: Quantifying the COVID-19 misinformation epidemic on Twitter. Cureus, 12(3), e7255.

Krishna, A., \& Thompson, T. L. (2019). Misinformation about health: A review of health communication and misinformation scholarship. American Behavioral Scientist. Advance online publication.

Kunda, Z. (1990). The case for motivated reasoning. Psychological Bulletin, 108(3), 480-498.

Lee, S. J., Sanders-Jackson, A., \& Tan, A. S. L. (2020). Effects of current and enhanced tobacco corrective messages on smokers' intention to quit smoking and intention to purchase cigarettes. Nicotine \& Tobacco Research, 22(4), 569-575.

Levy, A. G., Sonnad, S. S., Kurichi, J. E., Sherman, M., \& Armstrong, K. (2008). Making sense of cancer risk calculators on the Web. JGIM: Journal of General Internal Medicine, 23(3), 229-235.

Lewandowsky, S., Ecker, U. K. H., Seifert, C. M., Schwarz, N., \& Cook, J. (2012). Misinformation and its correction: Continued influence and successful 
debiasing. Psychological Science in the Public Interest, 13(3), 106-131.

Lyons, B., Merola, V., \& Reifler, J. (2019). Not just asking questions: Effects of implicit and explicit conspiracy information about vaccines and genetic modification. Health Communication, 34(14), 1741-1750.

Mahoney, L. M., Tang, T., Ji, K., \& Ulrich-Schad, J. (2015). The digital distribution of public health news surrounding the human papillomavirus vaccination: A longitudinal infodemiology study. JMIR Public Health and Surveillance, 1(1), e2.

Martinez, L. S., Hughes, S., Walsh-Buhi, E. R., \& Tsou, M. H. (2018). “Okay, we get it. You vape": An analysis of geocoded content, context, and sentiment regarding e-cigarettes on Twitter. Journal of Health Communication, 23(6), 550-562.

Matthews, A. K., Sellergren, S. A., Manfredi, C., \& Williams, M. (2002). Factors influencing medical information seeking among African American cancer patients. Journal of Health Communication, 7(3), 205-219.

Moran, M. B., Lucas, M., Everhart, K., Morgan, A., \& Prickett, E. (2016). What makes antivaccine websites persuasive? A content analysis of techniques used by anti-vaccine websites to engender anti-vaccine sentiment. Journal of Communication in Healthcare, $9(3), 151-163$.

Nyhan, B., \& Reifler, J. (2015). Does correcting myths about the flu vaccine work? An experimental evaluation of the effects of corrective information. Vaccine, 33(3), 459464.

Nyhan, B., Reifler, J., Richey, S., \& Freed, G. L. (2014). Effective messages in vaccine promotion: A randomized trial. Pediatrics, 133(4), e835-e842.

Oravec, J. A. (2000). Online medical information and service delivery: Implications for health 
education. Journal of Health Education, 31(2), 105-110.

Pluviano, S., Della Sala, S., \& Watt, C. (2020). The effects of source expertise and trustworthiness on recollection: The case of vaccine misinformation. Cognitive Processing. Advance online publication.

Qi, J., Trang, T., Doong, J., Kang, S., \& Chien, A. L. (2016). Misinformation is prevalent in psoriasis-related YouTube videos. Dermatology Online Journal, 22(11).

Randhawa, A. S., Babalola, O., Henney, Z., Miller, M., Nelson, T., Oza, M., Patel, C., Randhawa, A.S., Riley, J., Snyder, S., \& So, S. (2016). A collaborative assessment among 11 pharmaceutical companies of misinformation in commonly used online drug information compendia. The Annals of Pharmacotherapy, 50(5), 352-359.

Roozenbeek, J., \& van der Linden, S. (2019). Fake news game confers psychological resistance against online misinformation. Palgrave Communications, 5(1), 1-10.

Sangalang, A., Ophir, Y., \& Cappella, J. N. (2019). The potential for narrative correctives to combat misinformation. Journal of Communication, 69(3), 298-319.

Seymour, B., Getman, R., Saraf, A., Zhang, L. H., \& Kalenderian, E. (2015). When advocacy obscures accuracy online: Digital pandemics of public health misinformation through an antifluoride case study. American Journal of Public Health, 105(3), 517-523.

Smith, M. J., Ellenberg, S. S., Bell, L. M., \& Rubin, D. M. (2008). Media coverage of the measles-mumps-rubella vaccine and autism controversy and its relationship to MMR immunization rates in the United States. Pediatrics, 121(4), e836-e843.

Southwell, B. G., \& Thorson, E. A. (2015). The prevalence, consequence, and remedy of misinformation in mass media systems. Journal of Communication, 65(4), 589-595.

Steffens, M. S., Dunn, A. G., Wiley, K. E., \& Leask, J. (2019). How organizations promoting 
vaccination respond to misinformation on social media: A qualitative investigation. $B M C$ Public Health, 19(1), 1-12.

Sundstrom, B., Szabo, C., \& Dempsey, A. (2018). "My body. My choice": A qualitative study of the influence of trust and locus of control on postpartum contraceptive choice. Journal of Health Communication, 23(2), 162-169.

Swire-Thompson, B., \& Lazer, D. (2020). Public health and online misinformation: Challenges and recommendations. Annual Review of Public Health, 41, 433-451.

Tan, A. S. L., Lee, C., \& Chae, J. (2015). Exposure to health (mis)information: Lagged effects on young adults' health behaviors and potential pathways. Journal of Communication, 65(4), 674-698.

Teoh, D. (2019). The power of social media for HPV vaccination - Not fake news! American Society of Clinical Oncology Educational Book, 39, 75-78.

Thomas, J., Peterson, G. M., Walker, E., Christenson, J. K., Cowley, M., Kosari, S., Baby, K. E., \& Naunton, M. (2018). Fake news: Medicines misinformation by the media. Clinical Pharmacology and Therapeutics, 104(6), 1059-1061.

Thomas, R. J., Tandoc, E. C., \& Hinnant, A. (2017). False balance in public health reporting? Michele Bachmann, the HPV vaccine, and "mental retardation." Health Communication, 32(2), 152-160.

Vosoughi, S., Roy, D., \& Aral, S. (2018). The spread of true and false news online. Science, $359(6380), 1146-1151$.

Vraga, E. K., \& Bode, L. (2017). Using expert sources to correct health misinformation in social media. Science Communication, 39(5), 621-645.

Vraga, E. K., Kim, S. C., \& Cook, J. (2019). Testing logic-based and humor-based corrections 
for science, health, and political misinformation on social media. Journal of Broadcasting \& Electronic Media, 63(3), 393-414.

Walter, N., \& Murphy, S. T. (2018). How to unring the bell: A meta-analytic approach to correction of misinformation. Communication Monographs, 85(3), 423-441.

Walter, N., \& Tukachinsky, R. (2020). A meta-analytic examination of the continued influence of misinformation in the face of correction: How powerful is it, why does it happen, and how to stop it? Communication Research, 47(2), 155-177.

Wilkinson, T. A., Fahey, N., Shields, C., Suther, E., Cabral, H. J., \& Silverstein, M. (2012). Pharmacy communication to adolescents and their physicians regarding access to emergency contraception. Pediatrics, 129(4), 624-629.

Wood, T., \& Porter, E. (2019). The elusive backfire effect: Mass attitudes' steadfast factual adherence. Political Behavior, 41(1), 135-163.

Xu, Z., \& Guo, H. (2018). Using text mining to compare online pro- and anti-vaccine headlines: Word usage, sentiments, and online popularity. Communication Studies, 69(1), 103-122.

Zollo, F., Novak, P. K., Del Vicario, M., Bessi, A., Mozetič, I., Scala, A., Caldarelli, G., \& Quattrociocchi, W. (2015). Emotional dynamics in the age of misinformation. PloS One, 10(9), e0138740.

Żuk, P., Żuk, P., \& Lisiewicz-Jakubaszko, J. (2019). The anti-vaccine movement in Poland: The socio-cultural conditions of the opposition to vaccination and threats to public health. Vaccine, 37(11), 1491-1494. 